\title{
AFFINOR METRIC STRUCTURES AND THEIR PHYSICAL APPLICATIONS
}

\author{
Evgeniy S. Kornev
}

Kemerovo State University, Krasnaya Str. 6, Kemerovo, 650043 Russian Federation

Received May 17, 2016;

e-mail: q148@mail.ru

Accepted in revised form July 28, 2017;

Published December 30, 2016

\begin{abstract}
This work describes the fundamentals of the mathematical theory of affinor metric structures and physical problems where these structures are used. Affinor metric structure is defined as an arbitrary 1-form having a radical of arbitrary rank, a certain Riemannian metric and a special field of automorphisms of tangent spaces connecting the exterior differential of this 1-form and a metric. Affinor metric structures are a generalization of almost contact metric structures and Kahlerian structures with a precise fundamental 2-form. At the end of the work, applications of affinor metric structures in physics are described. The purpose of this article is to demonstrate possibilities of application of the theory of affinor metric structures when solving various mathematical and physical problems. In particular, the use of such structures to search for closed curves with a nonzero circulation of a vector field and construction of submanifolds on which the exterior differential of some 1-form induced by a vector field is non-degenerated. Methods of Riemann geometry and theories of differential forms and mathematical analysis on manifolds were used. The relevance of the subject matter is due to the most common case of statement of the problem for some 1-form with radical of arbitrary dimension. While in classic case, physics and geometry consider only 1 -forms with a zero radical.
\end{abstract}

Keywords: Affinor metric structure, radical of a 1-form, vector field effect on a curve

DOI: $10.21603 / 2500-1418-2016-1-2-34-40$

\section{INTRODUCTION}

The concept of an affinor metric structure arises as a natural generalization of almost contact and Kahlerian structures. However, in this paper we consider only the part of the mathematical theory of affinor metric structures, which is necessary to solve the following problem: Let us assume that $M$ is a simply connected manifold of dimension at least 3, $C$ is a closed curve in $M$ and $V$ is a vector field on a manifold $M$. Let $d r$ denote a vector field of infinitely small shift, and $\alpha$ denote a $1-$ form $(V, d r)$, where (.,.) is a scalar product of vector fields on $M$. If a manifold $M$ has dimension $n$, we obtain in local coordinates:

$$
\alpha=(V, d r)=V_{1} d x_{1}+\ldots+V_{n} d x_{n} .
$$

Let us consider a contour integral:

$$
\int_{C} \alpha=\int_{C}(V, d r) .
$$

Let us ask ourselves, when the integral (1) differs from zero for a given vector field $V$.

Due to simple connectivity of a manifold $M$ curve $C$ is always a boundary of some two-dimensional surface $S$. According to Stokes' formula we get:

$$
\int_{C} \alpha=\int_{S} d \alpha .
$$

Here $d \alpha$ is an external differential of a 1 -form $\alpha$. Typically, in physics, this surface integral is calculated using parametrization of a surface $S$. However, this approach does not allow to answer the question about vanishing of the integral (1) for arbitrary nonclosed 1-form $\alpha$. First of all, a surface integral can not always be calculated analytically, using a surface parameterization. Secondly, if numerically calculated integral is arbitrarily close to zero, it does not follow that it is exactly equal to zero. The theory of affinor metric structures allows to solve this problem by studying location of an original curve on a manifold as regard to two distributions of tangent subspaces generated by a $1-$ form $\alpha$ itself, chosen on $M$ by a Riemannian metric (scalar product) and some field of automorphisms of tangent spaces, called affinor. This set of objects will be called affinor metric structure. Affinor metric structure is a generalization of almost contact structures and almost Kahlerian structures with a precise fundamental 2-form. The main advantage of affinor metric structure is that 1 -form $\alpha$ is considered purely in general terms. It is not required that $d \alpha$ is a nondegenerated 2 -form, and there is no any requirement concerning parity of dimension of a manifold $M$. In the case of odd dimensional manifold $M$, affinor metric structure is exactly the almost contact metric structure. To date,

Please cite this article in press as: Kornev E.S. Affinor metric structures and their physical applications. Science Evolution, 2016, vol. 1, no. 2, pp. 34-40. doi: 21603/2500-1418-2016-1-2-34-40.

Copyright $(C)$ 2016, KemSU. This is an open access article distributed under the terms of the Creative Commons Attribution 4.0 International License (http:// creativecommons.org/licenses/by/4.0/), allowing third parties to copy and redistribute the material in any medium or format and to remix, transform, and build upon the material for any purpose, even commercially, provided the original work is properly cited and states its license. This article is published with open access at http:// scienceevolution.ru/ 
we have already obtained classification of invariant affinor metric structures for 3- and 4-dimensional homogeneous spaces (see. [1, 4]). Also, in [4], important results for spheres of arbitrary dimension were obtained. Moreover, in [3], a mathematical theory of affinor metric structures on vector bundles allowing the concept of an external differential of a 1-form is constructed and studied, and in [2], left-invariant affinor metric structures on Lie groups were studied.

In the first two sections of this paper, we study the necessary properties of a radical of a 1-form and affinor metric structures. Section 4 describes and studies sublagrangian submanifolds, which are also needed to solve the claimed problem. Finally, in Section 5 we give a definitive answer to the question raised and describe physical applications of this problem.

\section{Radical of a 1-form}

Let us assume that $M$ is a real manifold of dimension at least 3 and $\alpha$ is a global 1 -form on $M$. Let $I_{X} T=T(X,$. denote the inner product of a vector field $X$ and a covariant-type tensor field $T$.

Definition 2.1. Radical of a 1 -form $\alpha$ in a point $x \in M$ is a vector subspace $\operatorname{rad} \alpha_{x}=\left\{v \in T_{x} M\right.$ : $\left.I_{v} d \alpha_{x}=0\right\} .1$-form $\alpha$ is called regular if tangent subspaces $\operatorname{rad} \alpha_{x}$ have the same dimension at all points of $M$.

The definition directly implies the following facts:

1) Radical of a regular 1-form is a distribution of tangent subspaces on $M$;

2) 1 form is closed when, and only when its radical coincides with the whole tangent bundle TM;

3) External differential of a $1-$ form $\alpha$ is a symplectic 2-form on $M$ when, and only when $\operatorname{rad} \alpha=\{0\}$.

Let rad $\alpha$ denote a distribution of radicals of a regular 1 -form $\alpha$ on $M$ and by a radical rank we shall mean the rank of this distribution. A key result for a radical rank of regular 1-forms is the following theorem, which proof can be found in [2]:

Theorem 2.2. Let us assume that $\alpha$ is a regular nonclosed 1-form on a real manifold of dimension $n \geq 3$ and $r$ is a rank of distribution rada. Then:

1) If $n$ is even, then $r$ is even, too, and $0 \leq \underline{r} \leq n-2$.

2) If $n$ is odd, then $r$ is odd, too, and $1 \leq r \leq n-2$.

Remark 2.3. Let us note that a 1-form with a radical of rank 0 on the even-dimensional manifold $M$ defines a symplectic structure $d \alpha$ on $M$, and a 1 -form with a radical of rank 1 on the odd-dimensional manifold has a contact structure on $M$.

Let us assume that $D$ is an orthogonal complement to a distribution rad $\alpha$ with respect to some Riemannian metric on $M$. Distribution $D$ is called a working bundle, and $T M=D \oplus$ rad $\alpha$. Since the rank of a working bundle is a radical codimension and a difference of two arbitrary numbers of the same parity is always an even number, from Theorem 2.2 we obtain:

Corollary 2.4. Working bundle D has an even rank on the manifold of dimension of any parity.
Example 1. Let us consider an Euclidean space $R^{n}$ with coordinates $\left(x_{1}, \ldots, x_{n}\right)$. Let us define a 1 -form $\alpha=x_{1} d x_{2}$. Exterior differential of this 1 -form is as follows:

$$
d \alpha=d x_{1} d x_{2}
$$

This 1 -form is regular. Its radical consists of vectors with coordinates $\left(0,0, x_{3}, \ldots, x_{n}\right)$ and has a rank $n$ - 2 , and a working bundle consists of vectors with coordinates $\left(x_{1}, x_{2}, 0, \ldots, 0\right)$.

Example 2. Let us consider an Euclidean space $R^{4}$ with coordinates $\left(x_{1}, x_{2}, x_{3}, x_{4}\right.$,). Let us define a 1-form $\alpha=x_{1} x_{2} d x_{3}$. Exterior differential of this 1 -form is as follows: $\mathrm{T}$

$$
d \alpha=x_{2} x_{1} d x_{3}+x_{1} d x_{2} d x_{3} .
$$

With $x_{1}=x_{2}=0 d \alpha=0$ and $\operatorname{rad} \alpha=R^{4}$. For $x_{1} \neq 0$, $x_{2} \neq 0$, radical of a 1 -form $\alpha$ has a rank 2 and consists of vectors with coordinates $\left(y_{1},-\frac{x_{2} y_{1}}{x_{1}}, 0, y_{4}\right)$. Hence, it is clear that $\alpha$ is not a regular $1-$ form.

Let us assume that $\alpha$ is a regular non-closed $1-$ form on a manifold $M$ with a working bundle $D$, and distribution $D$ is holonomic. From the working bundle definition it follows that restriction of the exterior 2-form $d \alpha$ to the distribution $D$ is non-degenerated. If a working bundle $D$ is a holonomic distribution, then according to Frobenius theorem, there is a submanifold $Q:\left.D\right|_{Q}=T Q$ in $M$. In this case, we obtain that restriction $d \alpha$ to a submanifold $Q$ is a symplectic structure on $Q$, and $Q$ is a symplectic submanifold. Thus, we obtain:

Proposition 2.5. If there is a regular 1-form with a radical of rank $r \leq n-2$ and a holonomic working bundle on a manifold $M$ of dimension $n \geq 3$, then $Q$ contains a symplectic submanifold of dimension $n-r$.

From Corollary 2.4 it follows that the complex structure can be set on layers of a working bundle $D$ for any non-closed regular 1-form. Moreover, it is possible to request that such a complex structure preserves restriction of selected Riemannian metric to a working bundle $D$. This allows to turn to definition of affinor associated with a 1 -form.

\section{Affinor metric structures}

Let us assume that $M$ is a real manifold of dimension at least $3, \alpha$ is a regular non-closed 1 -form on $M, g$ is a Riemannian metric on $M$ and $D$ is a working bundle selected as an orthogonal complement to a radical of a $1-$ form $\alpha$.

Definition 3.1. Affinor associated with a 1 -form $\alpha$, is a continuous field of automorphisms $\Phi$ of tangent spaces, satisfying the conditions:

$$
\begin{gathered}
d \alpha(X, Y)=g(\Phi X, Y), X, Y \in C^{1}(T M), \\
G(\Phi X, \Phi Y)=g(X, Y), X, Y \in C^{1}(D) .
\end{gathered}
$$


We can obtain the following affinor properties immediately from the definition:

Proposition 3.2. Let us assume that $\Phi$ is an affinor associated with a regular non-closed 1-form $\alpha C^{1}$ with a working bundle $D$ on a manifold $M$. Then:

1) $\operatorname{ker} \Phi=\operatorname{rad} \alpha$;

2) $\left.\Phi^{2}\right|_{D}=-\mathrm{id}$, where id is an identity operator on $D$;

3) $d \alpha^{\circ} \Phi=d \alpha$

4) $d \alpha(X, \Phi X) \geq 0, X \in C^{1}(D)$.

Proof. Property 1 follows immediately from the definition of a radical of a 1-form $\alpha$ and definition 3.1. Let us prove a property 2 . For any $X, Y \in C^{1}(D)$ we obtain:

$$
\begin{gathered}
g(\Phi 2 X, Y)=d \alpha(\Phi X, Y)=-d \alpha(Y, \Phi X)= \\
=-g(\Phi Y, \Phi X)=-g(X, Y)
\end{gathered}
$$

Since Riemannian metric $g$ is non-degenerated on a working bundle $D$, we obtain $\Phi^{2} X=-X$.

Let us now prove a property 3 . For $X \in \operatorname{rad} \alpha$, equality $d \alpha(\Phi X, \Phi Y)=d \alpha(X, Y)$ is satisfied by virtue of property 1 and equalities from definition 3.1. For any $X, Y \in C^{1}(D)$, using property 2 and equality in definition 3.1, we obtain:

$$
\begin{gathered}
d \alpha(\Phi X, \Phi Y)=g\left(\Phi^{2} X, \Phi Y\right)=-g(X, \Phi Y)= \\
=-g(\Phi Y, X)=-d \alpha(Y, X)=d \alpha(X, Y) .
\end{gathered}
$$

Property 4 follows directly from the definition 3.1 . The proposition is proved.

Affinor $\Phi$ is easily described in a local basis of a tangent bundle TM. Let us assume that $\alpha$ is a regular 1form with a non-trivial radical on a manifold $M$ of dimension $n \geq 3, g$ is a Riemannian metric on $M$ and $e_{1}, \ldots, e_{\mathrm{n}}$ is a local orthonormal basis of a tangent bundle in a coordinate neighborhood of the point $x$. By Corollary 2.4 , the working bundle rank is equal to $2 l$, $l \geq 1$. Then in a local basis $e_{1}, \ldots, \mathrm{e}_{n}$, affinor matrix $\Phi$, associated with 1 -form $\alpha$ is as follows:

$$
\Phi=\left[\begin{array}{ll}
\frac{A_{2 l}}{0} & 0 \\
0 & 0
\end{array}\right],
$$

where $A$ is a skew-symmetric matrix of order $2 l$ with coefficients $a_{i j}=d \alpha\left(e_{i}, e_{j}\right)$, and matrix $\Phi$ is of the order $n$.

Now we can define affinor metric structure on an arbitrary manifold.

Definition 3.3. Affinor metric structure on a manifold $M$ is four objects $(\alpha, D, \Phi, g)$, where $\alpha$ is a non-closed regular 1 -form on $M, D$ working bundle on $M, \Phi$ is an affinor associated with a $1-$ form $\alpha$, and $g$ is a Riemannian metric on $M$, based on which affinor $\Phi$ and working bundle $D$ are defined.

Let us note that almost Kahlerian structure with a precise fundamental 2 -form on the even-dimensional manifold determines an affinor metric structure with the radical of rank 0 , and a contact metric structure on the odd-dimensional manifold is an affinor metric structure with the radical of rank 1 . An example of affinor metric structure is also an almost contact metric structure on an odd-dimensional manifold (see [1]).

Let us assume that $(\alpha, D, \Phi, g)$ is an affinor metric structure on a manifold $M$. Since Riemannian metric $g$ sets an isomorphism between tangent and cotangent bundles, there is a vector field $M$ on $\xi: \alpha=I_{\xi} g=g(\xi$, .). According to Riesz's theorem concerning linear functional, the vector field $\xi$ is unique. Let us call such vector field a characteristic vector field of affinor metric structure. Despite the fact that 1 -form $\alpha$ is regular, the characteristic vector field $\xi$ may vanish at points where $\alpha=0$. It is also obvious that $\alpha(\xi)=g\left(\xi, \xi=|\xi|^{2}\right.$. Generally, the characteristic vector field may either lie in rad $\alpha$ or not belong radical.

Definition 3.4. Affinor metric structure $(\alpha, D, \Phi$, $g$ ) with a characteristic vector field $\xi$ is called strict if $\xi \in \operatorname{rad} \alpha$.

The properties of strict affinor metric structures are studied in detail in [3]. Here we need only the following property of these structures:

Proposition 3.5. Let us assume that $(\alpha, D, \Phi, g)$ is a strict affinor metric structure on a manifold $M$ with dimension of not less than 3 . Then, working bundle $D$ lies in the core of a 1 -form $\alpha$.

Proof. Let us assume that $\xi$ is a characteristic vector field of strict affinor metric structure. It follows from the equality $\alpha(X)=g(\xi, X), X \in C^{1}(T M)$ that the vector field $\xi$ is orthogonal to ker $\alpha$ with respect to a metric $g$. From the definition of a working bundle $D$, it follows that distributions $D$ and $\operatorname{rad} \alpha$ are orthogonal with respect to metric $g$. Since codimension of the 1 -form core is equal to 1 and $\xi \in \operatorname{rad} \alpha$, radical of a 1 -form $\alpha$ may not lie in ker $\alpha$. We obtain that the working bundle $D$ lies in the orthogonal complement of the characteristic vector field $\xi$. But this orthogonal complement is ker $\alpha$. We obtain that $D \subseteq \operatorname{ker} \alpha$, and the proposition is proved.

For further purposes, we need an invariant definition of 1 -form external differential on a manifold. Let $X(f)$ denote the effect of the vector field $X$ on a function $F$, and $[X, Y]$ denote Lie bracket of vector fields $X$ and $Y$. Let us assume that $\alpha$ is a nontrivial 1 -form on a manifold $M$. Then, for any vector fields $X$ and $Y$ on $M$

$$
2 d \alpha(X, Y)=X(\alpha(Y))-Y(\alpha(X))-\alpha([X, Y]) .
$$

Proposition 3.6. Working bundle of a strict affinor metrical structure can not be a holonomic distribution.

Proof. Let us assume that $(\alpha, D, \Phi, g)$ is a strict affinor metric structure on a manifold $M$. Suppose that the working bundle $D$ is a holonomic distribution. By Frobenius theorem, a holonomic distribution is involutive. Owing to Proposition 3.5, for any $X, Y \in C^{1}(D)$ equation (2) takes the form:

$$
2 d \alpha(X, Y)=-\alpha([X, Y]) .
$$

Since the distribution $D$ is involutive, then $[X, Y] \in D \subseteq \operatorname{ker} \alpha$. We obtain that $\left.d \alpha\right|_{D} \equiv 0$. But this 
contradicts nondegeneracy of $\mathrm{d} \alpha$ on a working bundle. Consequently, a working bundle is nonholonomic, and the proposition is proved.

In the case where a working bundle $D$ of a strict affinor metric structure is completely nonholonomic, we obtain a pair $(D, g)$ is sub-Riemannian structure on a manifold $M$. Thus, we obtain:

Proposition 3.7. Any strict affinor metric structure on a manifold $M$ with a completely nonholonomic working bundle induces a sub-Riemannian structure on $M$.

Letusnotethattheconceptofaffinorisageneralization oftheconceptoforthogonalalmostcomplexstructure(see [5, Chapter 9]) for real manifolds of arbitrary dimension. If a working bundle $D$ of nonstrict affinor metric structure on a manifold $M$ is a holonomic distribution, then there is an integral submanifold $Q:\left.D\right|_{Q}=T Q$ in $M$, and restriction of affinor $\Phi$ to a submanifold $Q$ sets an almost complex structure on $Q$. It follows that existence of a holonomic working bundle of affinor metric structure on a manifold of arbitrary dimension implies existence of the almost complex submanifold, as in case of integrability of almost complex structure of complex submanifold on this manifold.

An important class of affinor metric structures is so-called K-affinor metric structures. Affinor metric structure $(\alpha, D, \Phi, g)$ is called K-affinor if its characteristic vector field $\xi$ is a Killing vector field, i. e. $\underset{\xi}{L} g=0$. Here $\underset{\xi}{L} g$ is a derivative of a Lie metric $g$ along the vector field $\xi$. Let us summarize the important results for K-affinor metric structures whose proof is given in [2] and [3].

Theorem 3.8. Suppose that $(\alpha, D, \Phi, g)$ is a $\mathrm{K}$-affinor metric structure with a characteristic vector field $\xi$ on a manifold $M$ of dimension at least 3. Then:

1) $\Phi=\nabla x i$, where $\nabla$ is a Levi-Civita coherence of Riemannian metric $g$;

2) For any vector field $X \in C^{1}(D)$, transversal to a characteristic vector field $\xi$, the sectional curvature in a two-dimensional direction $\{\xi, X\}$ is equal to 1 ;

3) If the metric $g$ is Einsteinian, its Ricci curvature and scalar curvature are positive;

4) If the metric $g$ is Einsteinian, then the manifold $M$ is compact and has a finite first fundamental group.

Theorem 3.9. Let us assume that $(\alpha, \mathrm{D}, \Phi, \mathrm{g})$ is a $\mathrm{K}$ affinor metric structure with a characteristic vector field $\xi, \nabla$ is a Levi-Civita coherence of metric $g$, and $L$ is a Lie derivative in the direction of a vector field $\xi$. Then the following conditions are equivalent:

1) K-affinor metric structure is strict;

2) The characteristic vector field $\xi$ is a geodesic vector field, i. e. $\nabla \xi \xi=0$;

3) $L \alpha=0$

In the case where the working bundle $D$ on a manifold $M$ of an affinor metrical structure is not holonomic, $M$ may contain submanifolds tangential to the distribution $D$, in which $d \alpha \equiv 0$. Since it is necessary to solve the problem stated in Section 1, let us turn to study of such submanifolds.

\section{Sublagrangian submanifolds}

Let us assume that $M$ is a real manifold of dimension $n,(\alpha, D, \Phi, g)$ is an affinor metric structure on $M$, and a working bundle $D$ is a nonholonomic distribution on $M$. From Theorem 2.2 it follows that with $n<5$ the distribution $D$ may only have a rank 0,1 or 2. By nontrivial submanifolds we shall mean manifolds $M$ of submanifold of dimension from 2 to $n-1$. If the rank of a working bundle $\leq 2$, $M$ does not contain any non-trivial submanifolds tangential to distribution $D$. Therefore, determination of sublagrangian submanifolds requires to consider manifolds of dimension at least 5 .

Definition 4.1. Sublagrangian submanifold for affinor metric structure $(\alpha, D, \Phi, g)$ on a manifold $M$ of dimension $\leq 5$ is a maximum nontrivial submanifold $Q: T Q \subset D$ and $\left.d \alpha\right|_{Q} \equiv 0$.

Recall that the definition of working bundle implies that the restriction $\mathrm{d} \alpha$ to the distribution $D$ is non-degenerated, and therefore, dimension of a sublagrangian submanifold cannot be the same as the rank of a working bundle. Furthermore, the definition 4.1 does not require that working bundle is nonholonomic distribution. Thus, the concept of a sublagrangian submanifold is defined for any affinor metric structure. In view of Corollary 2.4, working bundle always has an even rank. Definition 4.1 implies an initial assessment of dimension of sublagrangian submanifolds. If $2 l$ is a rank of working bundle, and $k$ is a dimension of sublagrangian submanifold, then $2 \leq k \leq 2 l-1$. In the case where the 1 -form radical rank $\alpha$ is 0 , sublagrangian submanifold is the classical sublagrangian submanifold for a symplectic structure $d \alpha$. Now let us obtain a more accurate estimate of dimension of sublagrangian submanifolds.

Theorem 4.2. Let us assume that $(\alpha, D, \Phi, g)$ is an affinor metric structure on a manifold $M$ of dimension $\leq 5$, and working bundle $D$ has the rank $2 l \leq 4$. Then, dimension of a sublagrangian submanifold for this affinor metric structure can not exceed 1 .

Proof. Let assume that $Q$ is a sublagrangian submanifold in $M$ and $k$ is dimension of submanifold Q. Using definitions 3.1 and 4.1 for any $X, Y \in C^{1}(T Q)$, we obtain:

$$
g(\Phi X, Y)=d \alpha(X, Y)=0
$$

We obtain that distributions $T Q$ and $\Phi T Q$ are

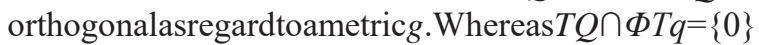
and $\operatorname{rank}(T Q)=\operatorname{rank}(\Phi T Q)$, we obtain:

$$
\operatorname{rank}(T Q \oplus \Phi T q)=2 k \leq \operatorname{rank}(D)=2 l .
$$

Finally, we obtain $k \leq 1$, and the theorem is proved.

Corollary 4.3. If the rank of a working bundle of affinor metric structure on a manifold $M$ is equal to 2 , 
then $M$ does not contain sublagrangian submanifolds for this affinor metric structure.

Remark 4.4. If $Q$ is a sublagrangian submanifold for an affinor metric structure $(\alpha, D, \Phi, g)$, then for any nontrivial submanifold $P \subset Q d \alpha \mid P \equiv 0$ is valid.

It is quite simple to obtain sublagrangian submanifolds for strict affinor metric structures. It is enough to take a maximal submanifold of appropriate dimension, tangential to a working bundle.

Theorem 4.5. Let us assume that $(\alpha, D, \Phi, g)$ is a strict affinor metric structure on a manifold $M$ and $Q$ is a maximum nontrivial submanifold $M$, tangential to a distribition $D$. Then $Q$ is a sublagrangian submanifold for this affinor metric structure.

Proof. In order to show that $Q$ is a sublagrangian submanifold, it is sufficiently to prove that $\left.d \alpha\right|_{Q} \equiv 0$. According to Proposition 3.5, TQ $\subset D \subseteq$ ker $\alpha$. Since the distribution $T Q$ is involutive, for any $X, Y \in C^{1}(T Q)$ we obtain $[X, Y] \in \operatorname{ker} \alpha$. Using equation (2) of section 3, we obtain:

$$
2 d \alpha(X, Y)=-\alpha([X, Y])=0
$$

Thus, determination 4.1 is satisfied, and the theorem is proved.

Corollary 4.6. Let us assume that $(\alpha, D, \Phi, g)$ is a strict affinor metric structure on a manifold $M$ with a working bundle of rank $2 l \leq 4$. Then dimension of any submanifold in $M$ tangential to a distribution $D$ may not exceed 1 .

Submanifolds of dimension 2 tangential to a working bundle are of particular interest. Let us assume that $M$ is a simply connected manifold of dimension at least 5 and $(\alpha, D, \Phi, g)$ is a strict affinor metric structure on $M$ with a working bundle of rank $2 l \leq 4$. Since any closed curve in a simply connected manifold is the boundary of a two-dimensional surface, we obtain that any closed curve on $M$ generates a two-dimensional submanifold. Let us assume that $C$ is a closed curve on $M$, tangential to working bundle $D$, such curves are called sublegendre curves and $S$ is a two-dimensional submanifold such that its boundary is $C$. Let us assume that $C$ is a vector tangential to a curve $C$. It's obvious that $\dot{C} \in C^{1}(D)$. The affinor properties obtained in Proposition 3.2 imply that the affinor $\Phi$ is invariant over the distribution $D$ and is a linear automorphism of a working bundle $D$. It follows that $\Phi \dot{C} \in C^{1}(D)$, vector fields $\dot{C}$ and $\Phi \dot{C}$ are transversal at all points where $\dot{C} \neq 0$, and submanifold $S$ is tangential to a distribution $D$. From Theorem 4.5 it follows that $S$ is contained in sublagrangian submanifold or coincides with it. Thus, we obtain the following result:

Theorem 4.7. Let us assume that $M$ is a simply connected manifold of dimension at least 5 , and $(\alpha$, $D, \Phi, g)$ is a strict affinor metric structure on $M$ with a working bundle of rank at least 4 . Then, $M$ contains a sublagrangian submanifold of dimension at least 2, and any closed curve on $M$ tangential to a distribution $D$, is a boundary of a two-dimensional submanifold contained in a sublagrangian submanifold.

Remark 4.8. From Theorem 4.7 it follows that any closed curve in a simply connected manifold tangential to a working bundle of a strict affinor metrical structure is always contained in sublagrangian submanifold.

Let us assume that $(\alpha, D, \Phi, g)$ is a nonstrict affinor metric structure with a characteristic vector field $\xi$. Let $\xi$ denote projection of a vector field $\xi$ on a working bundle $D$ and $\xi^{0}$ denote projection of a vector field $\xi$ on rad $\alpha$. Then, $\xi=\xi+\xi^{0}$, and for any vector field the following is valid $X$

$$
d \alpha(\xi, X)=d \alpha(\xi, X)+d \alpha\left(\xi^{0}, X\right)=d \alpha(\xi, X) .
$$

Thus, study of non-strict affinor metric structures is reduced to the case when characteristic vector field lies in a working bundle. Further, let us assume that $\xi \in C^{1}(D)$. Let $E$ denote a distribution $D \cap \operatorname{ker} \alpha$. A working bundle $D$ is decomposed into an orthogonal sum of a line generated by a characteristic vector field $\xi$ and distribution $E$. Let us assume that $S$ is a two-dimensional submanifold tangential to a distribution $E$. For any $X$, $Y \in C^{1}(T S)$ we obtain $X \in \operatorname{ker} \alpha, Y \in \operatorname{ker} \alpha,[X, Y] \in \operatorname{ker} \alpha$. Using equation (2) of section 3 , we obtain:

$$
2 d \alpha(X, Y)=-\alpha([X, Y])=0
$$

Whence $\left.d \alpha\right|_{S} \equiv 0$ and $S$ is contained in a sublagrangian submanifold.

Let us assume that $S$ is a two-dimensional submanifold tangential to a distribution $D$ so that the characteristic vector field $\xi$ is tangential to $S$. Since the codimension of the distribution $E$ in the distribution $D$ is always equal to 1 , there is a vector field $X: X \in C^{1}(E)$ in a manifold $S$. Then vector fields $\xi$ and $X$ form an orthogonal basis of the tangent bundle TS. Since $\alpha(X)=0$, from equation (2) in the section 3 , we obtain:

$$
2 d \alpha(\xi, X)=-X(\alpha(\xi))-\alpha([\xi, X]) .
$$

Let us assume that $L \alpha$ is a Lie derivative of a tensor

field $\alpha$ in the direction of a vector field $X$. There is an expression of the Lie derivative of a 1-form $\alpha$ through Lie brackets of vector fields (see [5, Chapter 1]):

$$
(\underset{x}{L} \alpha)(Y)=X(\alpha(Y))-\alpha([X, Y]),
$$

for any vector fields $X, Y$ is valid. Using this expression, we obtain:

$$
2 d \alpha(\xi, X)=-X(\alpha(\xi))-\alpha([\xi, X])=-(\underset{x}{L} \alpha) .
$$

It follows that $\left.d \alpha\right|_{S} \equiv 0$ when, and only when $(L \alpha)(\xi)=0$ on $S$. Thus, we obtain the following end $x$ result:

Theorem 4.9. Let us assume that $(\alpha, D, \Phi, g)$ is a affinor metric structure on a manifold $M$ of dimension at least 5 with a characteristic vector field $\xi: \xi \in C^{1}(D)$ and $S$ is a two-dimensional submanifold of a working bundle $D$. Then:

1) If a submanifold $S$ is tangential to a distribution $E=D \cap \operatorname{ker} \alpha$, then $\left.d \alpha\right|_{S} \equiv 0$; 
2) If the characteristic vector field $\xi$ is tangential to $S$ and $X$ is a vector field on $S$ orthogonal to $\xi$ with respect to a metric $g$, then $\left.d \alpha\right|_{S} \equiv 0$ when, and only when $(L \alpha)(\xi)=0$ on $\mathrm{S}$.

Now, we have reviewed all the properties of affinor metric structures needed to solve the problem posed in Section 1.

\section{Physical applications of affinor metric structures}

Let us assume that $M$ is a simply connected manifold of dimension at least $3, V$ is a vector field on $M$ and $g$ is a Riemannian metric on $M$. Then a global 1 -form $\alpha=$ $g(V$, , $)$ is defined on $M$. In local coordinates, the $1-$ form $\alpha$ looks like $g(V, d r)$, where $d r$ is a vector field of infinitely small shift. Let us define the following functional on the space of all closed curves:

$$
A_{V}: A_{V}(C)=\int_{C} \underline{\alpha},
$$

where $C$ is a closed curve on a manifold $M$. The meaning of this functional on a curve is used in various branches of physics. In mechanics $A_{V}(C)$ this is a work, done by a force that moves a body over a closed path $C$. In electrodynamics $A_{V}(C)$, this is a work of the electromotive force circulation in a closed conductor $C$. In the string theory, each elementary particle is represented as a curve of finite length in the Calabi-Yau space. For a particle identified with a closed curve $C, A_{V}$ (C) this is the effect of a vector field $V$, for example, a gravitational field on a particle. There is a need to determine curves for which a functional $A_{V}$ takes nonzero values.

Since the manifold $M$ is simply connected, for any closed curve $C$ given in an interval $[a, b]$, there exists a homotopy $F$ of a curve $C$ at the point $x_{0}$ :

$$
F(s, t): 0 \leq s \leq 1, a \leq t \leq b, F(1, t)=C(t), F(0, t)=x_{0}
$$

Then, the image of a two-dimensional space $[0,1] \times[a, b]$ under the effect of mapping $F$ is a two-dimensional surface in $M$ whose boundary is a curve $C$. Let $S$ denote this surface. According to Stokes' formula we obtain:

$$
\mathrm{A}_{\mathrm{v}}(\mathrm{C})=\int_{\mathrm{c}} \alpha=\int_{\mathrm{s}} d \alpha
$$

If the restriction of a 1 -form $\alpha$ to $S$ is a closed 1-form, then $A_{V}(C)=0$. If $\alpha$ is a non-closed regular 1 -form on $M$, then, defining by $D$ the orthogonal complement to a distribution rad $\alpha$ with respect to a metric $g$ and determining affinor $\Phi$ as in Section 3, we obtain an affinor metric structure $(\alpha, D, \Phi, g)$ with a working bundle $D$ and a characteristic vector field $\xi: \alpha(X)=g$ ( $\xi, X), X \in C^{1}(T M)$. Let us denote by $e_{1}$ and $e_{2}$ projections of vector fields $\frac{\partial}{\partial s}$ and $\frac{\partial}{\partial t}$ on a working bundle $D$, respectively. If $e_{1}=0$ or $e_{2}=0$, then $d \alpha$ $\left.\right|_{s} \equiv 0$ and $A_{V}(C)=0$. If $\mathrm{e}_{1}, \mathrm{e}_{2} \in$ ker $\alpha$, then, by virtue of clause 1 of Theorem $4.9,\left.d \alpha\right|_{s} \equiv 0$ and $A_{V}(C)=0$. Furthermore, from Theorem 4.7 it follows that with $\Phi \xi=0$ the surface $S$ is contained in a sublagrangian submanifold, and $A_{V}(C)=0$. Thus, we obtain:
Proposition 5.1. If a closed curve $C$ on a manifold $M$ is a boundary of a two-dimensional surface $S$, then $\alpha$ is a nonclosed regular $1-$ form on $M$, and one of the conditions is satisfied:

1) $S$ contains a vector field that lies in the radical of a 1 -form $\alpha$;

2) $S$ contains a couple of transversal vector fields, projections of which lie in the core of the 1-form $\alpha$;

3) 1-form $\alpha$ creates a strict affinor metric structure, then $A_{V}(C)=0$.

It remains to consider the case then $\Phi \xi \in C^{1}(T)$, and restriction of the characteristic vector field $\xi$ to $S$ is a vector field on $S$. In this case, there is a vector field $\xi^{1}: g\left(\xi, \xi^{1}\right)=\alpha\left(\xi^{1}\right)=0$ on $S$. From the clause 2 of Theorem 4.9 , it follows that

$$
\int_{s} d \alpha=\int_{w}\left(L_{\xi} \alpha\right)(\xi) d s d t=\int_{w} g\left(\Phi \xi, \xi^{1}\right) d s d t .
$$

Here $W$ is a range of variation of parameters $S$ and $t, \underset{x}{L}$ $\alpha$ is a Lie derivative of a 1 -form $\alpha$ in the direction of a vector field $X$. Note that if $\left(\Phi \xi, \xi^{1}\right)$, then $g\left(\Phi \xi, \xi^{1}\right)=$ $g(\xi, \xi) \geq 0$ (see Definition 3.1), and hence $A_{V}(C)>0$. In addition, in this case, the restriction of affinor $\Phi$ to $S$ is an almost complex structure on $S$. Since any almost complex structure on a two-dimensional manifold is always integrable (see [5, Chapter 9]), the restriction of affinor $\Phi$ is a complex structure on $S$. The physical meaning of the condition $\Phi \xi=\xi^{1}$ is that an angle between the vector field $V$ on a curve $C$ and the tangent vector field $\dot{C}$ does not exceed 90 degrees at any point of the curve. Let $X_{D}$ denote the projection of a vector field $X$ on a working bundle $D$. Now we obtain the following result:

Proposition 5.2. Let is assume that $(\alpha, D, \Phi, g)$ is an affinor metric structure on a manifold $M$ with a characteristic vector field $\xi \in C^{1}(D)$. If a closed curve $C$ is the boundary of a two-dimensional surface $S$, and there is a pair of orthogonal vector fields $X, Y: X_{D}$ $=\xi, \Phi X_{D}=Y_{D}$ on $S$, then $A_{V}(C)>0$.

Let us note that this proposal is not applicable in case where the function $g\left(\Phi \xi, \xi^{1}\right)$ takes both positive and negative values on the surface $S$. In this case, it requires a different criterion of variation from zero for the functional $A_{V}$. Let us assume that $C$ is a any closed curve defined in an interval $[a, b], S$ is a twodimensional surface, which boundary is a curve $C$, and restriction $d \alpha$ to $S$ is a non-degenerated exterior 2-form on $S$. In this case $S$ is a symplectic submanifold in $M$. Let us assume that $\{\varepsilon n\}$ is a infinitely small sequence such that $\varepsilon n>0, a+\varepsilon n<b-\varepsilon n$ for all real $n$. Let $d_{n}$ denote an interval $[a+\varepsilon n, b-\varepsilon n]$, and $\delta n$ - its length. It's obvious that $\lim _{\mathrm{n} \rightarrow \infty}\left\{\delta_{n}\right\}=b-a$.

Furthermore, there are sequences $\left\{\max _{n}\right\}$ and $\left\{\min _{n}\right\}$, where $\left.\max _{n}=\max _{t \in d} \alpha\left(\dot{C}_{t}\right)\right), \min _{n}=\min _{t \in d} \alpha\left(\dot{C}_{t}\right)$. 
We obtain:

$$
\min _{n} \delta n \leq \int d n \alpha(C(t)) d t \leq \max n \delta n .
$$

From here:

$$
\lim _{n \rightarrow \infty}\left\{\min _{n} \delta n\right\} \leq \int C \alpha \leq \lim _{n \rightarrow \infty}\{\max n \delta n\} .
$$

Since the sequence $\{\delta n\}$ is bounded, $\int C \alpha=0$ when, and only when the sequences $\left\{\max _{n}\right\}$ and $\left\{\min _{n}\right\}$ are infinitely small. Thus, we obtain the following criterion:
Proposition 5.3. If a closed curve $C$ on the manifold $M$ is the boundary of the two-dimensional surface $S, \alpha$ is a non-closed regular 1-form on $M$, and a restriction $d \alpha$ to $S$ is a symplectic structure on $S$, then $A_{V}(C)=0$ when, and only when $\lim _{\mathrm{n} \rightarrow \infty}\left\{\min _{n}\right\}=\lim _{\mathrm{n} \rightarrow \infty}\left\{\max _{n}\right\}=0$.

Remark 5.4. The criterion of Proposition 5.3 is applicable to any closed curve $C$, even on multiply connected manifold, when the curve $C$ is not a boundary of the twodimensional surface.

Thus, we have considered all the cases, and the problem posed at the beginning is solved.

\section{REFERENCES}

1. Calvaruso G. Three-dimensional homogeneous almost contact metric structures. Journal of Geometry and Physics, 2013, vol. 69, pp. 60-73.

2. Kornev E.S. Invariantnye affinornye metricheskie struktury na gruppakh Li [Invariant affinor metric structures on Lie groups]. Sibirskiy matematicheskiy zhurnal [Siberian Mathematical Journal], 2012, vol. 53, no. 1, pp. 107-123.

3. Kornev E.S. Affinornye struktury na vektornykh rassloeniyakh [Affinor structures on vector bundles]. Sibirskiy matematicheskiy zhurnal [Siberian Mathematical Journal], 2014, vol. 55, no. 6, pp. 1283-1296.

4. Kornev E.S., Slavolyubova Ya.V. Invariantnye affinornye i subkelerovy struktury na odnorodnykh prostranstvakh [Invariant affinor and sub-Kahlerian structures on homogeneous spaces]. Sibirskiy matematicheskiy zhurnal [Siberian Mathematical Journal], 2016, vol. 57, no. 1, pp. 51-63.

5. Kobayashi S., Namidzu K. Osnovy differentsial'noy geometrii [Foundations of Differential Geometry]. In 2 volumes, Moscow: Nauka Publ., 1981. 344 p.

\section{Evgeniy S. Kornev}

Cand.Sci.(Phys.-Math.), Leading engineer of the Mathematical Analysis Department, Kemerovo State University, Kemerovo, Russian Federation 